\title{
Revisiting the benefits of diversity representation in window operation models for building performance simulation
}

\author{
F. Tahmasebi ${ }^{1}$, and A. Mahdavi ${ }^{2}$ \\ 1 UCL Institute for Environmental Design and Engineering, London, United Kingdom \\ ${ }^{2}$ Department of Building Physics and Building Ecology, TU Wien, Vienna, Austria
}

\begin{abstract}
To explore the potential advantages of supplying occupant behavior models with inter-occupant diversity information, this study models the occupants' operation of windows in a monitored open-plan office at aggregate and individual levels. Subsequently, a calibrated energy model of the office area incorporates the developed models and multiple streams of monitored data to evaluate the predictive performance of the models and their contribution to enhance the reliability of building performance assessments. According to the results, individual window operation models outperformed the aggregate model in capturing the peak and variation of window operation across occupants in the free-running season, which resulted in a better assessment of thermal comfort. However, the individual models yielded an overestimation of peak heating demand, as compared with the benchmark value based on the actual window operations in a single year.
\end{abstract}

\section{INTRODUCTION}

The building simulation community has increased its efforts to reduce the gap between predicted and actual building energy use through probabilistic representations of occupant behaviour in buildings (Schweiker 2017). However, several studies have demonstrated that the use of existing occupant behaviour models involves considerable uncertainties and does not necessarily lead to a more reliable building performance assessment (e.g., Tahmasebi \& Mahdavi 2017; Gilani et al. 2017). Specifically, it is shown that without proper treatment of the diversity in occupants' behaviour, probabilistic occupancyrelated models fail to provide representative ranges of occupant behaviour possibilities as intended (O'Brien et al. 2017; Tahmasebi \& Mahdavi 2016). To address this issue, different approaches for inclusion of diversity in occupant behaviour modelling efforts have been examined (Reinhart 2004; Mahdavi 2015; O'Brien et al. 2017; Haldi et al. 2017). However, it has been also suggested that for specific cases, such as large open-plan offices (Gilani et al. 2017), a detailed treatment of occupants' diversity may not be beneficial. Consequently, further studies in this area are needed, as the potential benefits of supplying probabilistic occupant behaviour models with inter-occupant diversity information are not conclusively established. In this context, the current contribution revisits the problem through a case study. Thereby, the study investigates if modelling the inter-occupant diversity in operation of windows contributes to a more reliable performance analysis of an open-plan office area.

\section{METHOD}

\section{Overview}

The present study uses long-term monitored data on indoor and outdoor environment and state of windows in an office area to model the occupants' operation of windows with and without integration of inter-occupant variations in this regard. Subsequently, using the monitored window operation data along with a calibrated building performance model of the office area, the study explores two essential questions with regard to the use of stochastic window operation models: To which extent do the predictions of window operation models that disregard the behaviour diversity differ from the actual 
occupants' behaviour? To what degree does the consideration of occupants' behavioural diversity in building simulation contribute to better performance assessments?

\section{Building data}

The current study focuses on an office area with seven workstations in Vienna, Austria, where each occupant has access to one manually operable casement window (Figure 1). Six of these workstations are in an open-plan area. The building is not air-conditioned and it only uses a hydronic heating system to actively maintain thermal comfort in the cold season. In this office, the occupants' presence, state of windows and several environmental parameters (including indoor and outdoor air temperature) are monitored on a continuous basis. The study uses the monitored data from a calendar year (referred to as estimation period) to derive the window operation models. A separate set of data obtained from another calendar year (referred to as validation period) is used to evaluate the performance of the models.

\section{Window operation models}

To explore the research questions, the authors developed the following two types of window operation models based on the monitored data in the estimation period:

- Aggregate model (AGG), which was derived based on the data obtained from all occupants and windows;

- Individual models (W1-W7), which were derived based on the data obtained from each occupant and his/her associated window.

Each of the models consists of two logistic regression sub-models for estimation of window opening and closing probabilities. They provide the state transition probabilities for a Markov chain, based on which the states of windows are predicted for the validation period (for more details, see Tahmasebi \& Mahdavi 2018). Table 1 provides the estimated coefficients for the individual and aggregate window opening and closing models. Figure 2 illustrates slices through the response surface of individual and aggregate models at an outdoor temperature of $20^{\circ} \mathrm{C}$. Figure 3 depicts such slices at indoor temperatures of $20^{\circ} \mathrm{C}$ and $30^{\circ} \mathrm{C}$. 
Table 1: Estimated coefficients for logistic regression models of window opening and closing based on the individual windows (W1 - W7) and aggregate data.

\begin{tabular}{|c|c|c|c|}
\hline $\begin{array}{l}\text { Window } \\
\text { model }\end{array}$ & $\begin{array}{l}\text { Variables } \\
\text { and terms }\end{array}$ & $\begin{array}{c}\text { Opening } \\
\text { coefficients }\end{array}$ & $\begin{array}{l}\text { Closing } \\
\text { coefficients }\end{array}$ \\
\hline \multirow{4}{*}{$\begin{array}{l}\text { Individual } \\
\text { W1 }\end{array}$} & Intercept & -10.4233 & 16.6416 \\
\hline & $\theta_{\text {in }}$ & 0.0905 & -0.7013 \\
\hline & $\theta_{\text {out }}$ & 0.2047 & -0.5011 \\
\hline & Interaction & -0.0034 & 0.0186 \\
\hline \multirow{4}{*}{$\begin{array}{l}\text { Individual } \\
\text { W2 }\end{array}$} & Intercept & -12.2998 & 27.2410 \\
\hline & $\theta_{\text {in }}$ & 0.3135 & -1.1479 \\
\hline & $\theta_{\text {out }}$ & 0.1075 & -1.1022 \\
\hline & Interaction & -0.0032 & 0.0424 \\
\hline \multirow{4}{*}{$\begin{array}{l}\text { Individual } \\
\text { W3 }\end{array}$} & Intercept & -28.3577 & 7.9830 \\
\hline & $\theta_{\text {in }}$ & 0.9783 & -0.4323 \\
\hline & $\theta_{\text {out }}$ & 0.9343 & -0.3756 \\
\hline & Interaction & -0.0363 & 0.0144 \\
\hline \multirow{4}{*}{$\begin{array}{l}\text { Individual } \\
\text { W4 }\end{array}$} & Intercept & -31.2056 & 29.7625 \\
\hline & $\theta_{\text {in }}$ & 1.0775 & -1.3678 \\
\hline & $\theta_{\text {out }}$ & 0.4713 & -1.0668 \\
\hline & Interaction & -0.0195 & 0.0454 \\
\hline \multirow{4}{*}{$\begin{array}{l}\text { Individual } \\
\text { W5 }\end{array}$} & Intercept & -49.5078 & 15.5018 \\
\hline & $\theta_{\text {in }}$ & 1.9324 & -0.7982 \\
\hline & $\theta_{\text {out }}$ & 1.5071 & -0.6517 \\
\hline & Interaction & -0.0617 & 0.0283 \\
\hline \multirow{4}{*}{$\begin{array}{l}\text { Individual } \\
\text { W6 }\end{array}$} & Intercept & -22.4190 & 23.7542 \\
\hline & $\theta_{\text {in }}$ & 0.8031 & -1.0475 \\
\hline & $\theta_{\text {out }}$ & 0.3130 & -0.8246 \\
\hline & Interaction & -0.0123 & 0.0332 \\
\hline \multirow{4}{*}{$\begin{array}{l}\text { Individual } \\
\text { W7 }\end{array}$} & Intercept & -13.7355 & 25.3944 \\
\hline & $\theta_{\text {in }}$ & 0.4225 & -1.1495 \\
\hline & $\theta_{\text {out }}$ & 0.0343 & -0.9280 \\
\hline & Interaction & -0.0007 & 0.0374 \\
\hline \multirow{4}{*}{$\begin{array}{l}\text { Aggregate } \\
\text { (AGG) }\end{array}$} & Intercept & -10.6882 & 23.9665 \\
\hline & $\theta_{\text {in }}$ & 0.2187 & -1.0969 \\
\hline & $\theta_{\text {out }}$ & 0.2100 & -0.9172 \\
\hline & Interaction & -0.0052 & 0.0376 \\
\hline
\end{tabular}




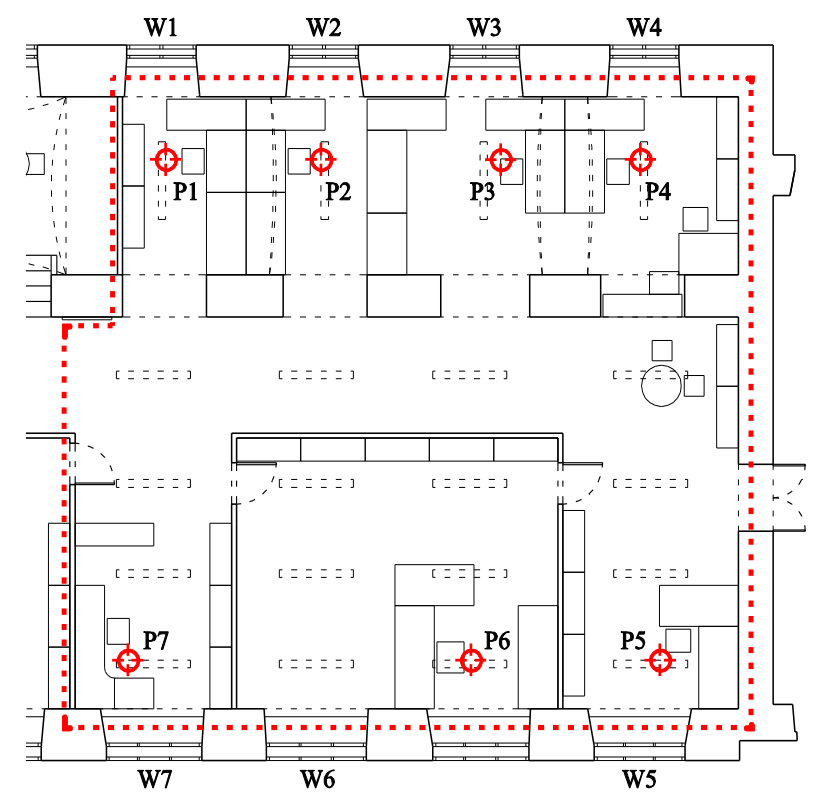

Figure 1: Schematic illustration of the office area, observed occupants and operable windows
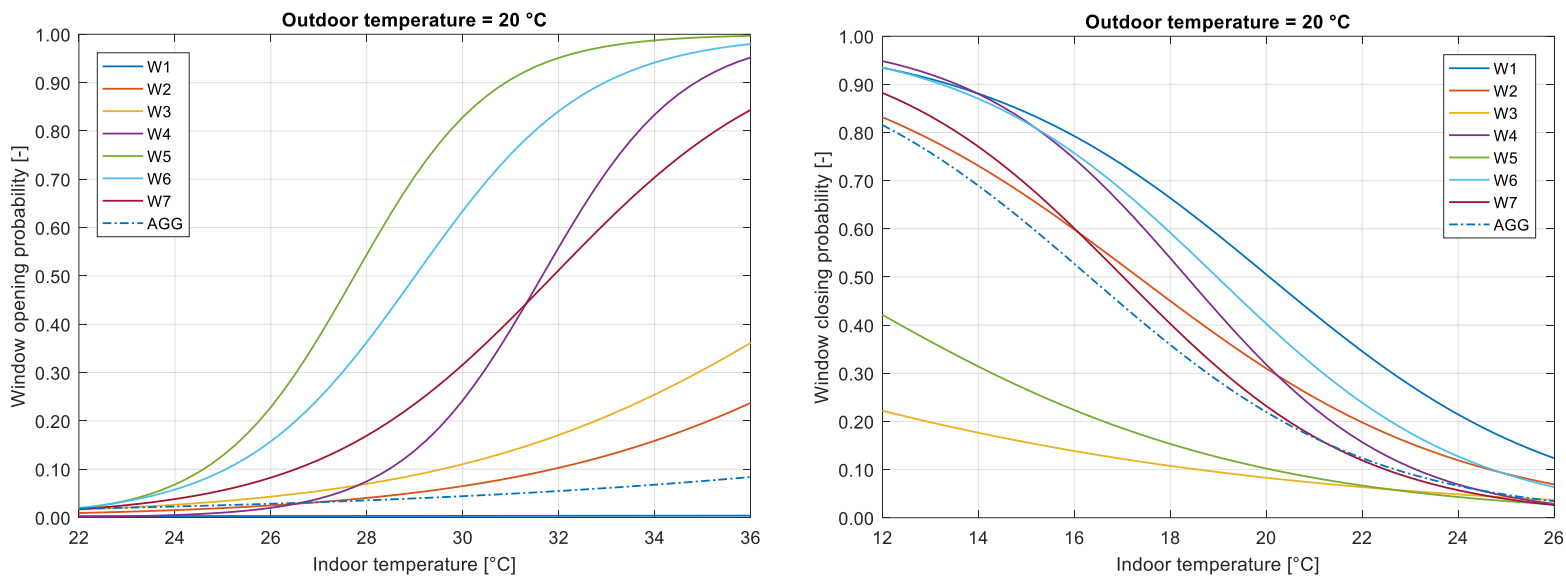

Figure 2: Slices through the response surface of individual and aggregate models for opening (left) and closing (right) of windows at an outdoor temperature of $20^{\circ} \mathrm{C}$. 

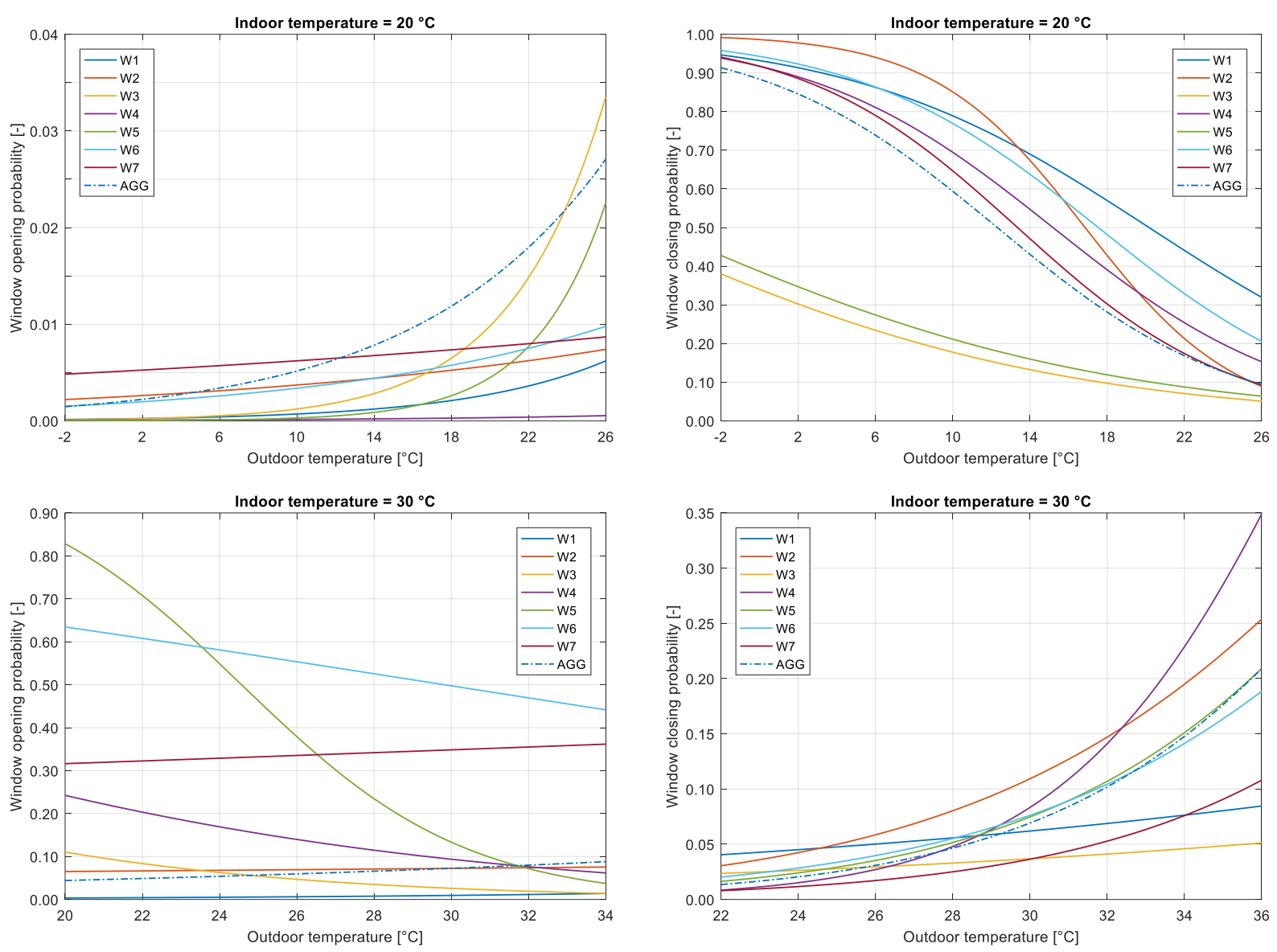

Figure 3: Slices through the response surface of individual and aggregate models for opening (left) and closing (right) of windows at indoor temperatures of $20^{\circ} \mathrm{C}$ (top) and $30^{\circ} \mathrm{C}$ (bottom).

Note that, in order to focus the investigation on the inter-occupant diversity representation, the current study adopted a rather simple approach to model window operation. Specifically, without conducting a comprehensive variable selection procedure, all the models only use indoor and outdoor temperatures as explanatory variables. However, the interaction term was added to the models to capture the effect of indoor temperature depending on outdoor temperature and vice versa. To the authors' knowledge, interaction of indoor and outdoor temperature has not been incorporated before in window operation models. This is, however, especially important in the present study, as in a number of extremely hot summer days in Vienna, natural ventilation cannot serve as a cooling measure and may not be preferable by occupants. As it can be seen in Figure 3, the developed models have captured this tendency: With an indoor temperature of $20^{\circ} \mathrm{C}$ and outdoor temperatures such as -2 to $26^{\circ} \mathrm{C}$, the probability of opening window increases with the rise of outdoor temperature (Figure 3, top left plot). However, in extreme summer conditions (for example with an indoor temperature of $30^{\circ} \mathrm{C}$ ), for a number of occupants, the probability of opening window is decreasing when the outdoor temperature rises (Figure 3, bottom left plot). A similar alternating pattern can be seen also in terms of estimated window closing probabilities (Figure 3, right plots).

Moreover, it should be noted that the models do no not differentiate between different occupancy phases (such as arrival, intermediate and departure). However, given the operational circumstances in the studied building, windows are assumed to be closed upon occupants' last departures.

\section{Diversity representation}

The present contribution adopts an ideal approach to represent inter-occupant diversity in window operation. Thereby, each individual model is used to predict the occupant, based on whom the model is 
developed. This strategy was due in part to the small number of occupants in the study. Moreover, it was considered consistent with the study's main aim, namely to explore the potential advantages of integrating diversity in occupant behaviour models.

\section{Office area calibrated simulation model}

The office area was modelled in the building energy simulation tool EnergyPlus 8.8.0. In the zoning scheme, the open-plan south and north-oriented spaces were separated from the central corridor. However, using the network-based multi-zone airflow model of EnergyPlus, the airflows across the external windows and the connected spaces were simulated. The constant input parameters governing airflow simulation in the EnergyPlus model (namely open windows discharge coefficient and closed windows air mass flow coefficient) were set based on a previous model calibration effort (Tahmasebi \& Mahdavi 2012).

The building calibrated simulation model served as a test bed for evaluation of window operation models with consideration of models' feedback, i.e. the impact of models' output (window states) on models' input (indoor temperature). The calibrated building model also made it possible to determine the impact of window operation (and use of different window operation models) on the estimation of building performance indicators. To this end, the monitored data streams of occupants' presence and use of lights and equipment in the validation year were fed into the model. In addition, the aggregate and individual window operation models were successively integrated into the building model using EnergyPlus runtime language. The study also benefited from a benchmark building model, which contained the actual states of windows based on the monitored data obtained in the validation period. Moreover, the building model was exposed to the outdoor environmental conditions in the validation period, using a weather data file generated from on-site weather station measurements. The measured dataset included outdoor air temperature, air humidity, atmospheric pressure, global horizontal radiation, diffuse radiation, wind speed, and wind direction. Lastly, due to the stochastic nature of the window operation models, the building model was simulated 50 times to obtain representative ranges of outputs.

It should be also noted that, an ideal unlimited heating system is set in the model to maintain the indoor temperature of different zones according to the measured indoor temperatures in the validation period, and to estimate the space heating demands. However, since such a system fully counteracts the impact of window openings, the building model is not strictly applicable to evaluation of window operation models in heating season. Therefore, for the purpose of the current study, the predictive performance of window operation models is only considered in the free-running season (from April 22 to September 25).

\section{Window operation evaluation metrics}

The following metrics serve to evaluate the window operation models in view of interval-by-interval equivalence of the predicted and monitored window states:

- TPR (True Positive Rate), as the proportion of actual open states, which are correctly predicted.

- FPR (False Positive Rate), denoting the proportion of actual closed states, which are wrongly predicted.

- TNR (True Negative Rate), denoting the proportion of actual closed states, which are correctly predicted.

- FNR (False Negative Rate) as the proportion of actual open states, which are wrongly predicted closed.

In addition, three indicators examine the dynamics and overall aspects of the predicted window operations, as follows:

- Overall fraction of open state, as the total window opening time divided by the observation time.

- Mean number of actions per day averaged over the observation time.

- Median open state duration, to capture the durations for which windows are left open. 
These indicators have been widely used in the studies pertaining to the evaluation of occupant behaviour models (e.g., Schweiker et al. 2010). However, in order to measure the models' ability to discriminate between occurrence and nonoccurrence of window openings and closings, the authors deployed a rather novel metric in this field, namely the coefficient of discrimination. This metric is suggested by Tjur (2009) in the context of logistic regression as an analogue to the coefficient of determination (R-squared) in ordinary regression models. The coefficient of discrimination (CoD) is calculated as follows:

$C o D=\bar{\pi}_{1}-\overline{\hat{\pi}}_{0}$

Where $\overline{\hat{\pi}}_{1}$ and $\overline{\hat{\pi}}_{0}$ denote the average of fitted values (estimated probabilities of window opening or closing), respectively, for the so-called successes (intervals, in which an opening or a closing action has been observed) and the failures (intervals, in which an opening or a closing action has not been observed). Similar to $\mathrm{R}$-squared in linear regression, coefficient of discrimination takes values between 0 and 1. A value of 0 corresponds to "no explanatory power" (all fitted values are equal), and a value of 1 corresponds to "perfect fit" (the fitted values coincide with the observations). This indicator offers two advantages in evaluation of occupant behaviour models: Firstly, it is obtained without conducting Monte Carlo simulation of the model (as it deploys the estimated probabilities and not the randomly-sampled states). Secondly, it does not require the inclusion of the models' feedback as it examines the models' predictions in a set of disconnected time intervals.

\section{Building performance indicators}

To capture the office area performance in heating and free-running seasons, the building energy model estimates the following indicators:

- Annual heating demand per floor area [kWh.m-2], which gives the total heating energy required in a year to maintain temperature set-points.

- Peak heating demand per floor area [W. $\left.\mathrm{m}^{-2}\right]$, which gives the maximum heating energy required in a 15-min interval to maintain temperature set-points.

- Time outside EN15251 comfort zone [\%], which denotes the fraction of occupied time in the free-running season, in which operative temperature is outside EN15251 Category III limits.

\section{RESULTS}

\section{Occupants' interactions with windows}

Table 2 provides the metrics obtained from interval-by-interval comparison of predicted and monitored window states of the entire office area in the free-running validation period. In addition, the models' ability to discriminate window opening and closing actions is captured with values of the coefficient of discrimination given in Table 3. This table also includes the coefficient of discrimination obtained from two widely-used existing window operation models developed by Rijal et al (2007) and Haldi and Robinson (2009) for reference.

Further aspects of window operation predictions, namely, overall fraction of open state, number of opening action and open state durations, are summarized in Table 4. It provides the observed and predicted values of the metrics for individual occupants/windows in the free-running season, together with the mean and standard deviation of the metrics across occupants. Note that, the values of the metrics in Table 2 and Table 4 are obtained from averaging the models' predictions in 50 Monte Carlo runs. To better compare the models' performance in capturing the variations in occupants' interactions with windows, Figure 4 shows the coefficient of variation of the metrics (as the ratio of standard deviation to the mean) obtained from the observations and predictions made by the aggregate and individual models. 


\section{Building performance indicators}

Table 5 gives the obtained building performance indicators, namely, annual and peak heating demands, and the percentage of occupied time outside EN15251 comfort limits. It includes the outputs of the benchmark model (with the monitored window operation data), along with the mean and standard deviation of the predictions provided by the building models equipped with the aggregate and individual window operation models. To put the models' performance in context, Table 5 also provides the results obtained from two simple window operation models. The first one assumes windows are open if indoor temperature is above $26^{\circ} \mathrm{C}$. The second one assumes windows are always closed.

To better interpret the obtained results, Figure 5 illustrates the relative error of the estimated building performance indicators as compared to the benchmark model. Note that, due to the very large overestimation of discomfort by the model with closed windows, the output of this model is not included in Figure 5. 
Table 2: Values of TPR, FPR, TNR, and FNR for aggregate and individual window operation models in the free-running validation period

\begin{tabular}{|c|c|c|c|c|}
\hline Model(s) & $\begin{array}{c}\text { TPR } \\
{[\%]}\end{array}$ & $\begin{array}{c}\text { FPR } \\
{[\%]}\end{array}$ & $\begin{array}{c}\text { TNR } \\
{[\%]}\end{array}$ & $\begin{array}{c}\text { FNR } \\
{[\%]}\end{array}$ \\
\hline Aggregate & 29.2 & 4.3 & 95.7 & 70.8 \\
\hline Individuals & 47.1 & 5.9 & 94.1 & 52.9 \\
\hline
\end{tabular}

Table 3: Values of Coefficient of Discrimination (CoD) for window opening and closing actions obtained from aggregate and individual window operation models in the validation period

\begin{tabular}{ccc}
\hline Model(s) & CoD opening [-] & CoD closing $[-]$ \\
\hline Aggregate & 0.008 & 0.082 \\
Individuals & 0.034 & 0.127 \\
Reference 1 (Rijal et al. 2007) & 0.044 & 0.008 \\
Reference 2 (Haldi and Robinson 2009) & 0.013 & 0.012 \\
\hline
\end{tabular}

Table 4: Observed and predicted mean values of fraction of open state, openings per day, and median open state duration for individual values along with inter-occupant mean and standard deviation of the metrics

\begin{tabular}{|c|c|c|c|c|c|c|c|c|c|}
\hline \multirow{2}{*}{ Window } & \multicolumn{2}{|c|}{ Overall fraction of open state [\%] } & \multicolumn{2}{|c|}{ Opening actions per day [d ${ }^{-1}$ ] } & \multicolumn{3}{|c|}{ Median open state duration [h] } \\
\cline { 2 - 9 } & Observed & $\begin{array}{c}\text { Aggregate } \\
\text { model }\end{array}$ & $\begin{array}{c}\text { Individual } \\
\text { models }\end{array}$ & Observed & $\begin{array}{c}\text { Aggregate } \\
\text { model }\end{array}$ & $\begin{array}{c}\text { Individual } \\
\text { models }\end{array}$ & Observed & $\begin{array}{c}\text { Aggregate } \\
\text { model }\end{array}$ & $\begin{array}{c}\text { Individual } \\
\text { models }\end{array}$ \\
\hline W1 & 2.5 & 5.5 & 0.5 & 0.18 & 0.45 & 0.07 & 2.00 & 2.38 & 1.46 \\
W2 & 7.8 & 6.5 & 4.8 & 0.46 & 0.48 & 0.50 & 3.75 & 2.57 & 1.62 \\
W3 & 3.6 & 7.5 & 8.8 & 0.18 & 0.52 & 0.55 & 4.00 & 2.68 & 3.13 \\
W4 & 10.8 & 6.8 & 7.3 & 0.48 & 0.54 & 0.52 & 4.75 & 2.31 & 2.56 \\
W5 & 8.8 & 7.2 & 19.7 & 0.50 & 0.56 & 1.02 & 3.25 & 2.43 & 3.43 \\
W6 & 9.3 & 5.0 & 11.7 & 0.50 & 0.43 & 1.04 & 2.75 & 1.99 & 1.52 \\
W7 & 17.8 & 6.7 & 13.6 & 0.69 & 0.52 & 0.83 & 4.13 & 2.50 & 3.37 \\
\hline Mean & 8.7 & 6.5 & 9.5 & 0.43 & 0.50 & 0.65 & 3.52 & 2.41 & 2.44 \\
SD & 4.7 & 0.9 & 5.8 & 0.17 & 0.04 & 0.32 & 0.86 & 0.21 & 0.83 \\
\hline
\end{tabular}

Table 5: Building performance indicators obtained from the building models equipped with monitored window operation (benchmark), aggregate and individual window operation models, and two simple non-probabilistic models

\begin{tabular}{|c|c|c|c|}
\hline $\begin{array}{c}\text { Window } \\
\text { operation } \\
\text { model(s) }\end{array}$ & $\begin{array}{c}\text { Annual } \\
\text { heating } \\
\text { demand } \\
\text { [kWh.m }\end{array}$ & $\begin{array}{c}\text { Peak } \\
\text { heating } \\
\text { demand } \\
\left.\text { [W. } \mathbf{m}^{-2}\right]\end{array}$ & $\begin{array}{c}\text { Time } \\
\text { outside } \\
\text { EN15251 } \\
\text { comfort } \\
\text { zone [\%] }\end{array}$ \\
\hline Benchmark & 64.7 & 89.3 & 5.6 \\
\hline Aggregate & $67.4 \pm 0.5$ & $95.5 \pm 15.5$ & $8.8 \pm 0.5$ \\
Individuals & $68.0 \pm 0.5$ & $111.0 \pm 20.8$ & $4.6 \pm 0.3$ \\
\hline Open if $\theta_{\text {in }}>26$ & 62.8 & 60.4 & 2.8 \\
Closed & 62.4 & 45.5 & 25.0 \\
\hline
\end{tabular}




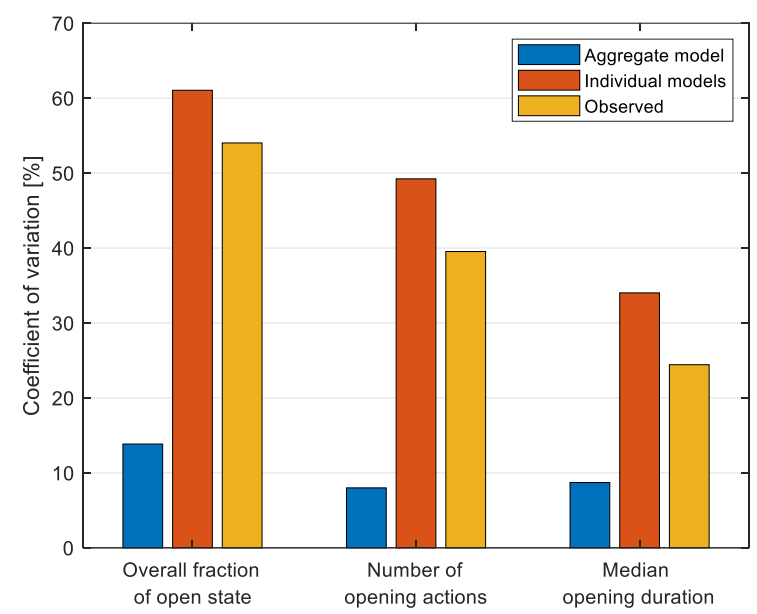

Figure 4: Coefficient of variation of the overall fraction of open state, number of opening actions, and median opening duration across occupants obtained from the observations, the aggregate and individual window operation models

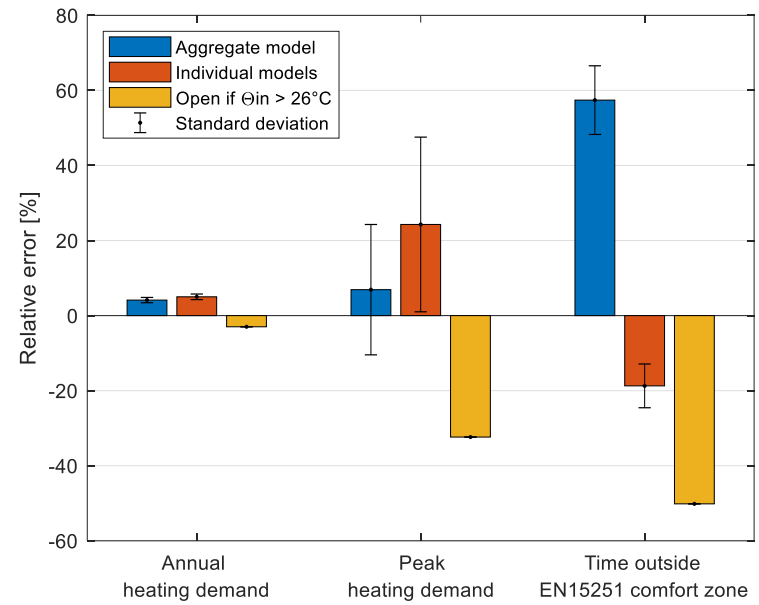

Figure 5: Relative error of the building performance indicators obtained from the building model with aggregate and individual window operation models as well as a simple rule-based model

\section{DISCUSSION}

\section{Occupants' interactions with windows}

The results provided in Table 2 indicate that individual window operation models outperform the aggregate model in correctly predicting open states (TPR of $47.1 \%$ versus $29.2 \%$ ), whereas their false predictions of open states are only slightly more frequent than those of the aggregate model (FPR of $5.9 \%$ versus $4.3 \%$ ). The individual models also generate fewer false predictions of closed states (FNR of $52.9 \%$ versus $70.8 \%$ ). Their performance in correctly predicting the closed states is very close to that of the aggregate model.

The low values of the coefficient of discrimination given in Table 3 for both aggregate and individual models suggest that their performance in this regard is far from satisfactory. However, the similar values obtained from the predictions by two reference models reveal that this poor performance is not exclusively attributable to the models developed in this study. In fact, this may be rather explained by the inherent limit to the extent to which one can predict the exact time of occupants' control-oriented actions. Nonetheless, the higher values of the coefficient of discrimination for the individual models, as compared with the aggregate model, suggests that the integration of diversity into the models has to some extent improved their ability in discriminating window opening and closing actions. 
Moreover, from the metrics provided for individual windows in Table 4, an interesting observation can be made concerning the overall fraction of open state: Even though each occupant's model is based on the data obtained from the same occupant in the estimation period, the individual models could not identify the occupant who kept the window open the longest. Based on observations, W7 shows the maximum overall fraction of open state with $17.8 \%$. However, in case of the individual models' predictions, the maximum overall fraction of open state belongs to W5 with $19.7 \%$. Nonetheless, and arguably more importantly, the individual models have outperformed the aggregate model in capturing the peak operation of individual windows. In fact, the aggregate model's lack of sensitivity to interoccupant diversity leads to the prediction that no occupant keeps the windows open longer than $7.5 \%$ of the time in free-running season. This has implications for the assessment of thermal comfort, which will be discussed in the next section.

As it can be seen from the mean values provided in Table 4, individual models do not show a better performance in predicting the average tendencies in terms of number of opening actions and opening duration. In fact, they even show a larger error in the average number of actions across occupants. However, the combination of an overestimation of the number of openings and an underestimation of the opening duration has resulted in this case in a better estimation of the overall fraction of open state. As for capturing the variations in occupants' interactions with windows, Figure 4 shows that the aggregate model has not captured the diversity among occupants. The individual models, however, have reproduced the variation of the metrics values across occupants with a slight overestimation.

\section{Building simulation results}

From Table 5, it can be seen that the aggregate and individual window operation models provide close estimations of the annual heating demand. Similarly, the non-probabilistic models, which disregard the possibility of window opening in heating season, provide reasonable estimates of annual heating demand. However, the non-probabilistic models fail to capture the peak heating demands resulting from occasional operation of windows in winter. In contrast, the aggregate and individual probabilistic models overestimate the peak values, which is more noticeable in case of individual models. Nonetheless, the benchmark peak heating demand (as one instance of possible annual peak demands in the building operation phase) is within one standard deviation of the mean predicted value of the aggregate model and very close to that of the individual models (Figure 5).

With regard to the estimation of thermal comfort in the free-running season (see Figure 5), the individual window operation models outperform the aggregate probabilistic model and the non-probabilistic ones. As mentioned before, even though the individual models could not identify the most 'active' windowoperating occupant in the validation period, they have done well in capturing the peak and the variations in the fraction of open state among occupants (see Table 4 and Figure 4). This has served the building model with individual window operation models to better estimate the occupants' thermal comfort in the free-running season (see Table 5 and Figure 5).

Delving further into this finding, one can hypothesize that, in a free-running open-plan office area with multiple occupants and windows, when few occupants open the windows (and, for example, enable cross ventilation) the rest of the occupants may not bother to open the windows anymore (perhaps, amongst other things, to avoid draught problem). Therefore, firstly, the occupants who open the windows more frequently may be different from time to time (as is the case in the estimation and validation periods in the present study). Secondly, due to the smaller number of 'active' occupants, as compared with the 'passive' ones (perhaps because in such an open-plan office operation of few windows suffice to change the indoor conditions), such adaptive actions may not be captured in models relying on averaged observations.

In this case study, the aggregate stochastic window operation model did not include the influence of 'active' occupants and therefore overestimated thermal discomfort. In contrast, the non-probabilistic model, which opens all the windows when indoor temperature exceeds $26^{\circ} \mathrm{C}$, underestimated the thermal discomfort, due to an overestimation of air change rate (which may not be even preferable for occupants). The results of the study suggested that, in the present case, the integration of inter-occupant 
behaviour diversity in window operation models could enhance the reliability of simulation-based thermal comfort analysis of an open-plan office area.

\section{CONCLUSION}

The study presented in this paper was limited to seven occupants in a specific office space in Vienna, Austria. The window operation models were developed and tested based on the data obtained from the same building. The study also examined an ideal case in representation of inter-occupant diversity, as the models estimated for individual occupants were used to predict the same occupants' interactions with windows. Nonetheless, the results suggest that integrating inter-occupant diversity in window operation models may be beneficial in terms of:

- Discrimination between windows' open and closed states throughout a long-term simulation;

- Discrimination between occurrence and nonoccurrence of window opening and closing actions;

- Estimation of the peak and variations of window operation across occupants, which cannot be captured by models derived from aggregate data;

- Assessing thermal comfort in an open-plan space, in which each occupant's adaptive actions could influence indoor environmental conditions of the whole space and thus influence other occupants.

With regard to the estimation of annual heating demand, the study did not show a benefit in using the aggregate or individual probabilistic models. As for the assessment of peak heating demand, the aggregate model showed a reasonable performance. The integration of inter-occupant diversity led to an overestimation of peak heating demand, as compared to the benchmark value resulting from the actual window operations in a single year.

Note that, while the present study points to the importance of occupants' behavioural diversity information in window operation models for specific building performance queries, it does not offer a generic and practical approach for the pervasive provision and systematic inclusion of such information in routine building performance simulation processes. Specifically, to provide effective simulation-based design support, occupant models would have to be based on statistically representative diversity information. As such information is currently unavailable, care should be taken in extrapolating from the results obtained from the few available empirical sources of data - such as what this study was based on. It should be also noted that a subsequent publication by the authors provides more details on the developed models and further explores their performance (Tahmasebi \& Mahdavi 2018).

\section{ACKNOWLEDGEMENTS}

The authors gratefully acknowledge that the research presented in this paper largely benefited from their participation in IEA-EBC Annex 79 (Occupant-Centric Building Design and Operation).

\section{REFERENCES}

Gilani, S., O'Brien, W., Gunay, B. 2018. Simulating occupants' impact on building energy performance at different spatial scales, Building and Environment 132, 327-337.

Haldi, F., Calì, D., Andersen, R.K., Wesseling, M., Müller D. 2017. Modelling diversity in building occupant behaviour: a novel statistical approach, Journal of Building Performance Simulation, 10:5-6, 527-544.

Haldi F., Robinson D., 2009. Interactions with window openings by office occupants. Building and Environment, 44(2009), pp 2378-2395, doi:10.1016/j.buildenv.2009.03.025.

Mahdavi, A. 2015. The inter-individual variance of the defining markers of occupancy patterns in office buildings: a case study, Proceedings of BS2015, 2243-2247.

O'Brien, W., H.B. Gunay, F. Tahmasebi, and A. Mahdavi. 2017. A preliminary study of representing the interoccupant diversity in occupant modelling, Journal of Building Performance Simulation, 10:5-6, 509-526.

Reinhart, C.F. 2004. Lightswitch-2002: a model for manual and automated control of electric lighting and blinds, Solar Energy 77(1) 15-28.

Rijal H.B, Tuohy P., Humphreys M.A., Nicol J.F., Samuel A., Clarke J., 2007. Using results from field surveys to predict the effect of open windows on thermal comfort and energy use in buildings. Energy and Buildings, 39 (2007), pp 823-836, doi:10.1016/j.enbuild.2007.02.003. 
Schweiker M., Haldi F., Shukuya M., Robinson D., 2012. Verification of stochastic models of window opening behavior for residential buildings, Journal of Building Performance Simulation, 5(1), 55-74.

Schweiker, M. 2017. Understanding occupants' behaviour for energy efficiency in buildings, Current Sustainable Renewable Energy Reports (2017) 4:8-14.

Tahmasebi, F., Mahdavi, A. 2012. Optimization-based simulation model calibration using sensitivity analysis, 7th Conference of IBPSA-CZ, Brno, Czech Republic.

Tahmasebi, F., Mahdavi, A. 2016. An inquiry into the reliability of window operation models in building performance simulation, Building and Environment 105 (2016), 343-357.

Tahmasebi, F., Mahdavi, A. 2017. The sensitivity of building performance simulation results to the choice of occupants' presence models: a case study. Journal of Building Performance Simulation, 10:5-6, 625-635.

Tahmasebi, F., Mahdavi, A. 2018. On the utility of occupants' behavioural diversity information for building performance simulation: an exploratory case study, Energy and Buildings (2018), DOI: 10.1016/j.enbuild.2018.07.042.

Tue Tjur, 2009. Coefficients of Determination in Logistic Regression Models - A New Proposal: The Coefficient of Discrimination, The American Statistician, 63:4, 366-372, DOI: 10.1198/tast.2009.08210. 